\title{
Simulation of Spherocylinder Particle Transport Phenomena using Discrete Element Method
}

\author{
Yanyong Angklomkleaw ${ }^{1}$, Thongchai Rohitatisha Srinophakun ${ }^{2 *}$, and Sirapong Tengpavadee ${ }^{3}$ \\ ${ }^{1}$ Chemical Engineering Practice School, King Mongkut's University of Technology Thonburi, Bangkok 10500, Thailand \\ ${ }^{2}$ Department of Chemical Engineering, Kasetsart University, Bangkok 10900, Thailand \\ ${ }^{3}$ School of bio-chemical engineering and technology, Sirindhorn International Institute of Technology, Thammasat University, \\ Pathum Thani 12120, Thailand \\ * Corresponding author's email: fengtcs [AT] hotmail.com
}

\begin{abstract}
Spherocylinder particle motion is simulated in three dimensions using a combined approach of discrete element method and computational fluid dynamics (DEM-CFD). This work is carried out since there are several limitations to use the spherical model for representing the transport phenomena of other particle geometries. Two thousands of particles in a $150 \times 150 \times 400 \mathrm{~mm}$ bed are studied for the particle motion, the effect of superficial gas velocity and the bed configuration on the particle temperature distribution and drying rate. The initial temperature and moisture content of particles is $298 \mathrm{~K}$ and 0.35 , the temperature of the gas is $331.5 \mathrm{~K}$. Heat transfer from gas to a particle is estimated using the modified Ranz-Marshall equation where the influence of surrounded particle on the heat transfer is considered. The results from random particle packing under the gravity are used as the initialize step of the simulations. From the simulation, the results are fairly realistic in particle motion, particle temperature distribution and the drying rate. The results demonstrate that the particle temperature distribution and the drying rate increase with the inlet gas velocity. For the number of feed inlets, the increasing number of feed inlet improves particle movement and particle temperature distribution. However, the drying rate of particles in 4 gas inlets bed decreases from the drying rate of particles in 2 feed inlets bed. This phenomenon comes from the air fed through each nozzle has a shorter period.
\end{abstract}

Keywords - Computational Fluid Dynamic (CFD)/ Distinct Element Method (DEM)/ Particle Motion / Spherocylinder Particle.

\section{INTRODUCTION}

Spouted beds are gas-particle contactors where the gas is passed through a single nozzle at the center of a conical or flat base. The solid particles, initially at rest driven by the fluid drag force and the inter-particle forces from neighbor particles, start to move and exhibit complex flow patterns. The spouted bed provides the direct contact between the fluid and solid particles to improve mass and heat transfer between particle and fluid; it can be applied to many processes such as drying of granular materials and catalyst [1]. This technique is suitable for the Geldart's type D particle which is the large size and high-density particle.

Unfortunately, it is difficult to observe and measure gas and particle dynamics without disturbing the flow field since the particle and fluid motions occur inside the bed. Therefore, a numerical model such as "Discrete Element Method" was developed to describe the behavior of solid particles in the bed. The Discrete Element Method (DEM) established by Cundall and Strack [2] is a numerical model capable of describing the mechanical behavior of discs and spheres assemblies. The motions of individual particles and gas flow are obtained by solving Newton's second law of motion and Navier-Stokes equation, respectively. The method is based on the use of an explicit numerical scheme in which the interaction of the particles is monitored contact by contact and the motion of the particles is modeled particle by particle. Previously on the studies of transport phenomena of particles, the studies focused only on spherical particles. This is a large limitation since such models can not determine many exciting phenomena. Therefore, a new three-dimensional discrete element simulation method that can handle spherocylinder particles is developed from the previous work to improve accuracy. Also, the study of heat and mass transfer for spherocylinder particle will be carried out. 


\section{THEORY}

\subsection{Spouted Bed}

The spouted bed was invented by Gishler and Mathur [4] for drying of grain. In a spouted bed, continuous circulation flow of particles is observed. Particles flow downward in the annulus with a small velocity component to the center line. Then the particles are driven upward by the high-speed gas. In each cycle, mixing of solids occurs mainly within the two dilute regions, spout, and fountain.

The hydrodynamic features of a spouted bed are significantly different from those of a conventional fluidized bed in that a systematic cyclic movement is imparted to the solids which travel upward through a dilute axial zone and upwards through a small orifice at the center of the conical base rather than distributing it evenly across the bed.

\subsection{Distinct Element Method}

From Newton's second law of motion, the total force acting on the particle must be known to calculate its motion. The equations for total force acting on an individual particle can be divided into translation and rotation respectively as shown in Equation (1) and (2)

$$
\begin{gathered}
m \frac{d v}{d t}=f_{c}+f_{d}+f_{f}+m g \\
I \frac{d \omega}{d t}=T
\end{gathered}
$$

Since the model deals with individual spherocylinder particle, it is necessary to consider the collisions of particle-toparticle and particle-to-wall. This work uses the two endpoints of each spherocylinder particle to determine the particle's position in the spouted bed. The knowledge of analytic geometry, such as the distance between two points and the distance from a point to a line is used to determine collision point. The schematic diagram of particle-particle collision for spherocylinders is in Fig.1, and particle-wall collision is presented in Fig.2.

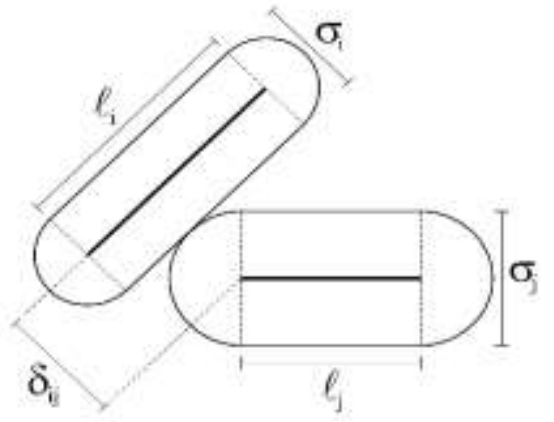

Figure 1: Schematic diagram of the particle-particle collision for spherocylinders [3]

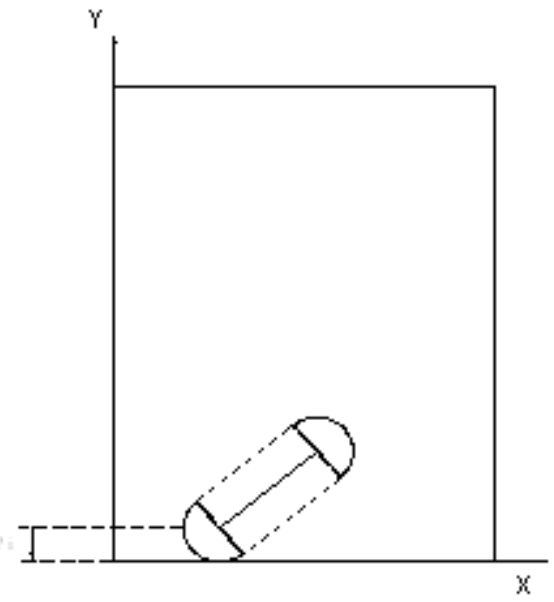

Figure 2: Two-dimensional representation of possible contacts between a spherocylinder and wall 
For a pair of spherocylinders, the collision occurs when the shortest distance between their shafts ( $\delta \mathrm{ij})$ is less than the sum of their radius. Considering the bed wall in Fig.2, the collision occurs if the distance between the wall and the center line of the particle is less than the particle's radius. An algorithm of Abreu et al. [3] with the modified version of Pournin et al. [5] for parallel particles was used to calculate the shortest distance between two shafts of the spherocylinders, in general. If the particles in contact are parallel, the shortest distance comes from

$$
\delta_{i j}=\left|r_{i j}\right|^{2}-\mid u_{i}\left[\left.r_{i}\right|^{2}+\left\{\max \left[0, \lambda_{i j}-\left(1_{i}-1_{j}\right) / 2\right]\right\}^{2}\right.
$$

Where $r_{i j}$ is the vector connects the center of shaft $i$ to the center of shaft $j$, that is, $r_{i j}=r_{j-} r_{i} . u_{i}$ is the unit vector in its orientation direction of particle $\mathrm{i}$

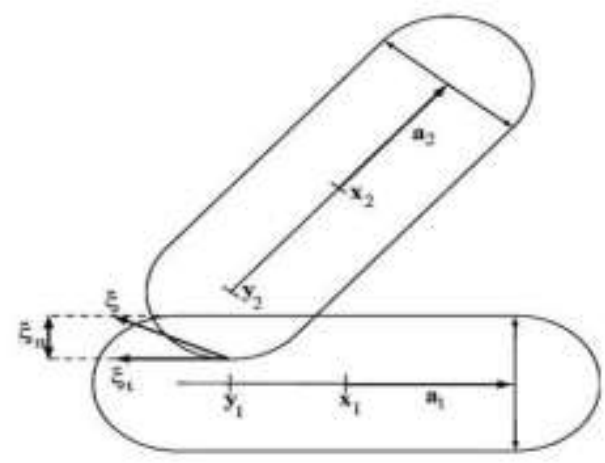

Figure 3: Schematic diagram of particle-particle collision for spherocylinders

For the general cases, the shortest distance is calculated from the distance between two points $y_{1} \in \varphi_{\mathrm{i}}$ (shaft of particle $i$ ) and $y_{2} \in \varphi_{\mathrm{j}}$ (shaft of particle $j$ ). In the calculation, $y_{1}=x_{1}+s a_{1}$ and $y_{2}=x_{2}+t a_{2}$ for $\mathrm{s}, t \in[-1,1]$. It is also assumed that these shafts are non-degenerate or $a_{1} \neq 0$ and $a_{2} \neq 0$. Now the values of $x_{1}, a_{1}, x_{2}, a_{2}$ are known and $\mathrm{s}$ and $\mathrm{t}$ can be obtained from $\mathrm{t}=\alpha\left(\frac{\mathrm{bd}-\mathrm{ae}}{\mathrm{h}}\right)$ and $\mathrm{s}=\alpha\left(\frac{-\mathrm{bt} *-\mathrm{d}}{\mathrm{a}}\right)$. If $\left(\frac{-\mathrm{bt}-\mathrm{d}}{\mathrm{a}}\right)$ is not $\in[-1,1]$, compute $\mathrm{t}=\alpha\left(\frac{-\mathrm{bs}-\mathrm{e}}{\mathrm{c}}\right)$. Where $\mathrm{a}=a_{1}^{2}, \mathrm{~b}=-$ $a_{1} a_{2}, \mathrm{c}=a_{2}^{2}, \mathrm{~d}=a_{1}\left(x_{1}-x_{2}\right), \mathrm{e}=-a_{2}\left(x_{1}-x_{2}\right), \mathrm{h}=\mathrm{ac}-\mathrm{b}^{2}, \alpha$ is defined as:

$$
\alpha(u)=\begin{gathered}
-1 \text { if } u \leq-1 \\
u \text { if } u \in(-1,1) \\
1 \text { if } u \geq 1
\end{gathered}
$$

For the shortest distance between a spherocylinder and wall, it is adapted from Abreu et al.'s[3] work as shown in Equation (5).

$$
\Delta i=\min \left[\left|\mathrm{r}_{\mathrm{i}}-\left(\mathrm{I}_{\mathrm{i}} / 2\right) \mathrm{u}_{\mathrm{i}}\right|,\left|\mathrm{r}_{\mathrm{i}}-\left(\mathrm{I}_{\mathrm{i}} / 2\right) \mathrm{u}_{\mathrm{i}}\right|\right]
$$

If a collision takes place on the particles $\mathrm{i}$ and $\mathrm{j}$, as shown in Fig.3. For these contact forces, it is necessary to define parameters which will be used to calculate the normal vector of the contact area. First of all, it is essential to find two points $y 1 \in \varphi \mathrm{i}$ (shaft of particle i) and y2 $\in \varphi \mathrm{j}$ (shaft of particle j). For the case when the shafts $\varphi \mathrm{i}$ and $\varphi \mathrm{j}$ of particle $\mathrm{i}$ and particle $\mathrm{j}$ are in general position, the pair $(\mathrm{y} 1, \mathrm{y} 2)$ is the same as in the calculation of the shortest distance between two spherocylinders. When shaft $\varphi \mathrm{i}$ and shaft $\varphi \mathrm{j}$ are parallel, it has a lot of pairs (y1, y2). Start with p: $\varphi \mathrm{i} \rightarrow \mathrm{L}$ be the projection of $\varphi \mathrm{i}$ on the line $\mathrm{L}$ supporting segment $\varphi \mathrm{j}$. If $\mathrm{p}(\varphi \mathrm{i}) \cap \varphi \mathrm{j}=\emptyset$, y2 will be the point on shaft $\varphi \mathrm{j}$ closest to $\mathrm{p}(\varphi \mathrm{i})$ and $y 1$ is the point on shaft $\varphi \mathrm{i}$ whose image under $\mathrm{p}$ is the point of $\mathrm{p}(\varphi \mathrm{i})$ closest to shaft $\varphi \mathrm{j}$. If $\mathrm{p}(\varphi \mathrm{i}) \cap \varphi \mathrm{j} \neq \varnothing$, y2 is the point which is in center of $\mathrm{p}(\varphi \mathrm{i}) \cap \varphi \mathrm{j}$ and $\mathrm{y} 1$ as the point on shaft $\varphi \mathrm{i}$ that projects on $\mathrm{y} 2$. After obtaining the value of $\mathrm{y} 1$ and $\mathrm{y} 2$, the unit vector "normal" to the contact can be defined as:

$$
u_{n}=\frac{y_{2}-y_{1}}{\left\|y_{2}-y_{1}\right\|}
$$

$u_{\mathrm{n}}$ is the normal vector to the tangent plane of spherocylinder. The application points of forces $f_{i \rightarrow j}$ and $f_{j \rightarrow i}$ are defined as the intersections of $\left[y_{1}, y_{2}\right]$ with the boundaries of particle $\mathrm{i}$ and $\mathrm{j}$, they are $c_{2}=y_{2}-r_{2} u_{n}$ and $c_{1}=y_{1}+r_{1} u_{n}$, respectively. The relative velocity $v_{r}$ at the contact is the velocity of $c_{2}$ to $c_{1}$,

$$
\mathrm{v}_{\mathrm{r}}=\mathrm{v}_{2}-\mathrm{v}_{1}-\left(\mathrm{y}_{2}-\mathrm{x}_{2}-\mathrm{r}_{2} \mathrm{u}_{\mathrm{n}}\right) \times \omega_{2}+\left(\mathrm{y}_{1}-\mathrm{x}_{1}+\mathrm{r}_{1} \mathrm{u}_{\mathrm{n}}\right) \times \omega_{1}
$$


The overlap $\xi$ is the solution of the differential equation

$$
\dot{\xi}=-\mathrm{v}_{\mathrm{r}}
$$

$\xi$ is assumed to be 0 when the contact begins. The normal and tangential overlaps are the projections of $\xi$ on vector $u_{n}$ and on the plane perpendicular to vector $u_{n}, \xi_{\mathrm{n}}=\mathrm{v}_{\mathrm{r}}\left[\mathrm{u}_{\mathrm{n}}\right.$ and $\xi_{\mathrm{t}}=\xi-\left(\xi \square \mathrm{u}_{\mathrm{n}}\right) \mathrm{u}_{\mathrm{n}}$. In addition, $u_{n}$ is assumed to be constant during a contact. Then the time derivatives for the normal and tangential overlaps are

$$
\begin{gathered}
\xi_{\mathrm{n}}=\mathrm{v}_{\mathrm{r}} \square \mathrm{u}_{\mathrm{n}} \\
\dot{\xi}_{\mathrm{t}}=-\mathrm{v}_{\mathrm{r}}+\left(\mathrm{v}_{\mathrm{r}} \square \mathrm{u}_{\mathrm{n}}\right) \mathrm{u}_{\mathrm{n}}
\end{gathered}
$$

From the previous work, the value of $\dot{\xi}$ is equal $-\mathrm{v}_{\mathrm{r}}\left[\mathrm{u}_{\mathrm{n}}\right.$ but from the theory of vector and the examination from the simulation results, it should be the positive value. Equation (6), (7) and (9) give

$$
\xi_{\mathrm{n}}=\mathrm{r}_{2}-\mathrm{r}_{1}-\left\|\mathrm{y}_{2}-\mathrm{y}_{1}\right\|
$$

The normal overlap is defined as the length of the line connecting $c_{1}$ and $c_{2}$. The normal force fn and the tangential force $\mathrm{ft}$ can be calculated by a force-overlap relation. The calculation of the contact force is the linear viscoelastic model.

$$
\begin{aligned}
& \mathrm{f}_{\mathrm{n}}=\left(\mathrm{k}_{\mathrm{n}} \xi_{\mathrm{n}}+\mathrm{c}_{\mathrm{n}} \dot{\xi}_{\mathrm{n}}\right) \mathrm{u}_{\mathrm{n}} \\
& \mathrm{f}_{t}=\left(\mathrm{k}_{t} \xi_{t}+\mathrm{c}_{t} \dot{\xi}_{t}\right) \mathrm{u}_{t}
\end{aligned}
$$

The magnitude of the tangent force is calculated and verified with the permitted formula $\left\|f_{t}\right\|>\mu\left\|f_{n}\right\|$ where $\mu$ is the coefficient of friction at the contact point. If the slip occurs, $f_{t}$ is replaced by $f_{t}=\mu \frac{\left\|f_{n}\right\|}{\left\|f_{t}\right\|} f_{t}$. For particle-wall collision, if the collision takes place at an end $c$, let $d$ be normal to the wall and passing through $c$. The application points of the forces acting on the particle and the wall are defined as the intersections of $d$ with the boundary of the particle and with the wall. The contact force is calculated similarly to the contact between spherocylinders except for mass, velocity. The mass of the wall is assumed to be infinity with $0.0 \mathrm{~m} / \mathrm{s}$ for velocity. When overlaps exist at both ends, for example, if the particle is parallel to the wall, the contact is modeled by two forces. The other treatments are the same as particle-toparticle collision except that the values of e and $\mu$ for the particle-to-wall collision are different from the above ones. For each contact, applying the physical contact model gives expressions for the forces acting on the particles and the walls. Forces $f_{l}, \ldots, f n$ act on a spherocylinder $i$ with mass $m$, the moment of inertia $I$. If force $f_{i}$ has application point $c_{i}$, the particle motion can be calculated as

$$
\begin{gathered}
m \ddot{x}=m g+f_{1} \ldots+f_{n} \\
I \dot{\omega}=\left(c_{1}-x\right) \times f_{1}+\ldots+\left(c_{n}-x\right) \times f_{n}
\end{gathered}
$$

In the model, the positions of the particles are computed and updated individually from Equations (14) and (15) in each time step. For spherocylindrical particles, the moment of inertia is expressed in a diagonal matrix with referential $x$, $y$, and $z$, whose diagonal elements $I_{x x}, I_{y y}$, and $I_{z z}$ are given by

$$
\begin{gathered}
\mathrm{I}_{\mathrm{xx}}=\frac{1}{48} \pi \rho \sigma^{2} \mathrm{I}^{3}+\frac{3}{64} \pi \rho \sigma^{4} \mathrm{I}+\frac{1}{60} \pi \rho \sigma^{5}+\frac{1}{24} \pi \rho \sigma^{3} \mathrm{I}^{2} \\
\mathrm{I}_{\mathrm{yy}}=\mathrm{I}_{\mathrm{xx}} \\
\mathrm{I}_{z z}=\frac{1}{32} \pi \rho \sigma^{4} \mathrm{I}+\frac{1}{60} \pi \rho \sigma^{5}
\end{gathered}
$$

The fluid drag force is the force between particle and fluid. It depends on both the relative velocity of fluid and particle and the presence of other surrounding particles. This force represents the interaction between the fluid and the particles and is given by

$$
\mathrm{f}_{\mathrm{f}, \mathrm{i}}=\frac{\beta}{(1-\varepsilon)}\left(\mathrm{u}-\mathrm{v}_{\mathrm{p}}\right) \times \mathrm{V}_{\mathrm{p}}
$$

Where $v_{p}$ and $V_{p}$ are the averaged velocity and volume of each particle; respectively. The coefficient $\beta$ is given by Ergun's equation [6] for the dense region and the equation derived by Wen and $\mathrm{Yu}$ [7] for the dilute region; 


$$
\begin{gathered}
\beta=\frac{\mu_{\mathrm{f}}(1-\varepsilon)}{\mathrm{d}^{2} \varepsilon}\left[150(1-\varepsilon)+1.75 \mathrm{Re}_{\mathrm{p}, \mathrm{i}}\right] \quad \varepsilon \leq 0.8 \\
\beta=\frac{3}{4} \mathrm{C}_{\mathrm{D}} \frac{\mu_{\mathrm{f}}(1-\varepsilon)}{\mathrm{d}_{\mathrm{p}}{ }^{2}} \varepsilon^{-2.7} \operatorname{Re}_{\mathrm{p}, \mathrm{i}} \quad \varepsilon \leq 0.8 \\
\operatorname{Re}_{\mathrm{p}}=\frac{\mathrm{d}\left|\mathrm{u}_{\mathrm{f}}-\mathrm{u}_{\mathrm{p}}\right| \rho_{\mathrm{f}}}{\mathrm{u}_{\mathrm{f}}}
\end{gathered}
$$

$\mathrm{C}_{\mathrm{D}}$ can be calculated from the equation below [8].

$$
\begin{aligned}
& \operatorname{Re}_{\mathrm{p}} \leq 1000 \\
& \mathrm{c}_{\mathrm{D}}=\frac{24}{\operatorname{Re}} \frac{\mathrm{d}_{\mathrm{a}}}{\mathrm{d}_{\mathrm{n}}}\left[1+\frac{0.15}{\sqrt{\mathrm{c}}}\left(\frac{\mathrm{d}_{\mathrm{A}}}{\mathrm{d}_{\mathrm{n}}} \operatorname{Re}_{\mathrm{p}}\right)^{0.687}\right]+\frac{0.42\left(\frac{\mathrm{d}_{\mathrm{A}}}{\mathrm{d}_{\mathrm{n}}}\right)^{2}}{\sqrt{\mathrm{c}}\left[1+4.25 \times 10^{4}\left(\frac{\mathrm{d}_{\mathrm{A}}}{\mathrm{d}_{\mathrm{n}}} \operatorname{Re}_{\mathrm{p}}\right)^{-1.16}\right]} \\
& \operatorname{Re}_{\mathrm{p}}>1000 \\
& \mathrm{C}_{\mathrm{D}}=0.9
\end{aligned}
$$

\subsection{Computational Fluid Dynamics}

The mathematical equations used in the calculation of fluid flow are governed by three fundamental principles: mass conservation, momentum conservation, and energy conservation. Most of the equations are usually partial differential equations named governing equations (e.g. the Navier-Stokes equations for incompressible Newtonian fluids), which cannot be solved analytically. The Computational Fluid Dynamics (CFD) is used to solve a numerical solution to the governing equations of fluid flow. The finite volume method is used in the simulation in many mathematical simulation calculations of flow fields. The flow domain is divided into cells, the size of cell should be smaller than the macroscopic motion of the bubbles in the fluidized bed but larger than the particle size for achieving the local average value of the fluid properties such as velocity and pressure. An equation of continuity;

$$
\frac{\partial \varepsilon}{\partial t}+\nabla \square(\varepsilon u)=0
$$

An equation of momentum transfer in $\mathrm{x}, \mathrm{y}$, and $\mathrm{z}$-direction;

$$
\begin{gathered}
\frac{\partial}{\partial t}(\varepsilon u)+\frac{\partial}{\partial x}(\varepsilon u u)+\frac{\partial}{\partial y}(\varepsilon v u)+\frac{\partial}{\partial z}(\varepsilon w u)=-\frac{\varepsilon}{\rho} \frac{\partial P}{\partial x}+\frac{K_{x}}{\rho} \\
\frac{\partial}{\partial t}(\varepsilon v)+\frac{\partial}{\partial x}(\varepsilon u v)+\frac{\partial}{\partial y}(\varepsilon v v)+\frac{\partial}{\partial z}(\varepsilon w v)=-\frac{\varepsilon}{\rho} \frac{\partial P}{\partial y}+\frac{K_{y}}{\rho} \\
\frac{\partial}{\partial t}(\varepsilon w)+\frac{\partial}{\partial x}(\varepsilon u w)+\frac{\partial}{\partial y}(\varepsilon v w)+\frac{\partial}{\partial z}(\varepsilon w w)=-\frac{\varepsilon}{\rho} \frac{\partial P}{\partial z}+\frac{K_{z}}{\rho}
\end{gathered}
$$

The equation of motion;

$$
\begin{gathered}
\frac{\partial\left(\rho_{f} \varepsilon u\right)}{\partial t}+\nabla \square\left(\rho_{f} \varepsilon u u\right)=-\varepsilon \nabla \rho-F \\
\mathrm{~F}=\frac{\sum_{\mathrm{i}=\mathrm{n}}^{\mathrm{n}} \mathrm{f}_{\mathrm{f}, \mathrm{i}}}{\Delta \mathrm{V}}
\end{gathered}
$$

\subsection{Drying of solids}

Drying is the process to remove a small amount of liquid from the solid material. Typically, the moisture content of a solid is expressed as the moisture content by weight of bone-dry material in the solid. Solids can be classified as no hygroscopic capillary-porous media, hygroscopic-porous media and colloidal (nonporous) media. The drying behavior of solids can be characterized by measuring the moisture content loss as a function of time as shown in Fig.4. After a short period in which the feed is heated to the wet-bulb temperature, the graph becomes linear, then toward the horizontal and finally level off. Most of the drying rate period is horizontal, indicating that the drying rate is constant. During this period, the solid is so wet that a continuous film of water exists over the entire drying surface. In the absence of heat transfer by radiation or by conduction through direct contact of the solid with a hot surface, the temperature of the solid 
during the constant-rate period is the wet-bulb temperature of the air. The drying rate can be divided into three different periods as follows.

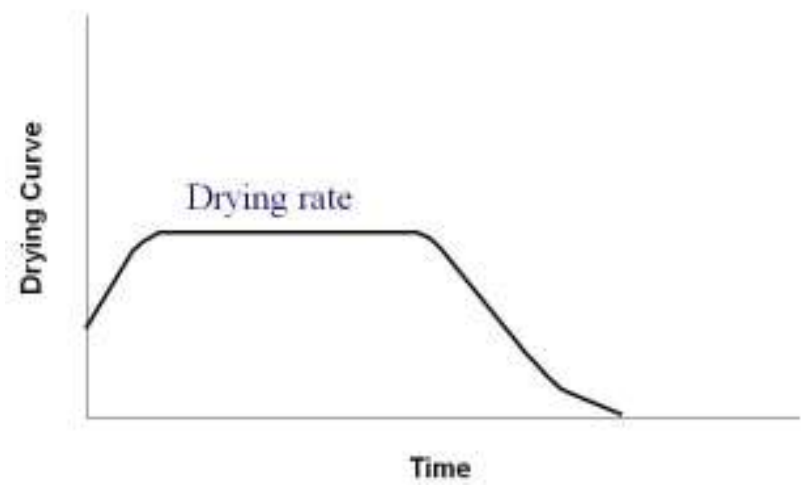

Figure 4: Typical plots of drying rate vs. drying time

1. Heating-up period In this period, the particle is heated up to the wet-bulb temperature. There is no condensation and drying, so only heat transfer governs the process.

$$
h\left(T_{f}-T_{p}\right) S_{p}=V c_{p p} \rho_{p} \frac{d T_{p}}{d t}
$$

2. Constant rate period. Drying rate in this period is controlled only by heat transfer from the drying medium to the particle, which provides the enthalpy of evaporation for evaporating the moisture.

$$
h\left(T_{f}-T_{p}\right) S_{p}=-\frac{d_{w}}{d t} \lambda_{w}
$$

3. Falling rate period It is assumed that the particle is small enough so that its temperature is uniform. It is further assumed that the radiant heat transfer is unimportant.

$$
h\left(T_{f}-T_{p}\right) S_{p}=V c_{p p} \rho_{p} \frac{d T_{p}}{d t}-\frac{d_{w}}{d t} \lambda_{w}
$$

In the simulation, the heating-up period was used for heating continuously the particles and then the constant rate period was used for the particles that were heated until the remaining wet-bulb temperature. The method used to calculate the temperature of the fluid is similar to the technique used for fluid motion.

$$
\frac{\partial\left(c_{p f} p_{f} \varepsilon T_{f}\right)}{\partial t}+\nabla\left\lceil\left(c_{p f} p_{f} \varepsilon u T_{f}\right)=\frac{6(1-\varepsilon)<\eta\left(T_{p}-T_{f}\right)>}{d_{p}}\right.
$$

Where $\mathrm{h}=$ Heat transfer coefficient estimated by Li and Mason (2000); $\mathrm{S}_{\mathrm{p}}=$ External surface area of a particle.

$$
\begin{gathered}
\operatorname{Re}_{p} \leq 200 \\
\mathrm{Nu}=2.0+0.6 \varepsilon^{3.5} \operatorname{Pr}^{1 / 3} \operatorname{Re}_{\mathrm{p}}{ }^{1 / 2} \\
200<\operatorname{Re}_{p} \leq 1500 \\
\mathrm{Nu}=2.0+0.5 \varepsilon^{3.5} \operatorname{Pr}^{1 / 3} \operatorname{Re}_{\mathrm{p}}{ }^{1 / 2}+0.02 \varepsilon^{3.5} \operatorname{Pr}^{1 / 3} \operatorname{Re}_{\mathrm{p}}{ }^{4 / 5} \\
\operatorname{Re}_{p}>1500 \\
\mathrm{Nu}=2.0+0.000045 \varepsilon^{3.5} \operatorname{Re}^{1.8} \mathrm{p} \\
\mathrm{Nu}=\frac{\mathrm{hd}}{\lambda_{\mathrm{f}}} \\
\operatorname{Pr}=\mathrm{c}_{\mathrm{pf}} \mu_{\mathrm{g}} / \lambda_{\mathrm{f}}
\end{gathered}
$$




\section{PROGRAMMING}

1. Particles are spherocylinder with the same particle size.

2. No hygroscopic capillary-porous media in spherocylinder shape is assumed for the particles.

3. The particle-to-particle adhesion forces are neglected because they are significantly small when compared to other forces. Moreover, buoyancy force is very small due to the very high density of particle compared that of fluid so it can be neglected.

4. Particles are supposed to have a soft sphere interaction expressed by a Hookean linear spring, a dashpot and a friction slider with Coulomb's friction law.

5. Gas is inviscid except when considering the fluid drag force on the particle.

6. The size of the particle is small enough, so its temperature is uniform.

7. Physical properties such as thermal conductivity, density, and viscosity of the gas and heat capacity of the particle are constant.

8. The correlation between $\mathrm{Li}$ and Mason [9] is applied to calculate the gas to particle heat transfer coefficient.

9. Particle-to-particle heat transfer is neglected because of short contact time.

10. Heat loss through the bed wall is neglected.

11. The radiation heat transfer is unimportant.

12. The effect particle shape on the stiffness can be neglect (Stiffness of sphere and spherocylinder is the same).

13. The drying process concerned in this work is only in constant rate period.

Table 1: Parameters of the simulation

\begin{tabular}{ll}
\hline Particle & $2 \mathrm{~mm}$ \\
Diameter $(\sigma)$ & $4 \mathrm{~mm}$ \\
Length $(1)$ & 2.000 \\
Aspect Ratio* $(\varphi)$ & $2700 \mathrm{~kg} \mathrm{~m}^{-3}$ \\
The density of bulk particle & $800 \mathrm{~N} \mathrm{~m}^{-1}$ \\
Stiffness & $0.35 \mathrm{~kg} \mathrm{~kg}^{-1}$ (Dry basis) \\
Initial moisture content & $1000 \mathrm{~kg} \mathrm{~m}^{-3}$ \\
Density of moisture content & $962 \mathrm{~J} \mathrm{Kg} \mathrm{K}^{-1}$ \\
Heat capacity & 2000 \\
Number of particles & \\
Gas & $0.970 \mathrm{~kg} \mathrm{~m}^{-3}$ \\
Density & $2.09 \times 10^{-5} \mathrm{~N} \mathrm{~m} \mathrm{~K}^{-2} \mathrm{~S}^{-1}$ \\
Viscosity & $1065.9 \mathrm{~J}(\mathrm{~kg} \mathrm{~K})^{-1}$ \\
Heat capacity & $0.03116 \mathrm{~W} \mathrm{(m} \mathrm{K})^{-1}$ \\
Heat conductivity & $331.48 \mathrm{~K}$ \\
Dry-bulb temperature & $313.15 \mathrm{~K}(30 \%$ Relative humidity) \\
Wet-bulb temperature & \\
Others & \\
Number of orifices & 2 \\
Number of cell in X, Y, and Z & $15,10,15$ \\
in direction & \\
\hline Aspect ratio $=$ length/diameter & \\
\hline
\end{tabular}

\section{RESULTS AND DISCUSSION}

\subsection{Program Testing}

Following cases are used to test the implementation of several force algorithms, in free motion and single contacts to test the accuracy of DEM code.

\subsubsection{Falling Particles}

This case shows a gravity force that all particles are set at the same height and then fall with gravity. They fall to the bottom of the wall and bounce with force from Newton's law. The viscous damping force is included in this validation to 
consume excess energy. The rebounded height decreases with time because of the damping force. There are two particles with different orientation in this study to study the effect of orientation on the motion of the particle. The first particle is laid horizontally in the $\mathrm{X}-\mathrm{Z}$ plane whereas the other is in a vertical direction.
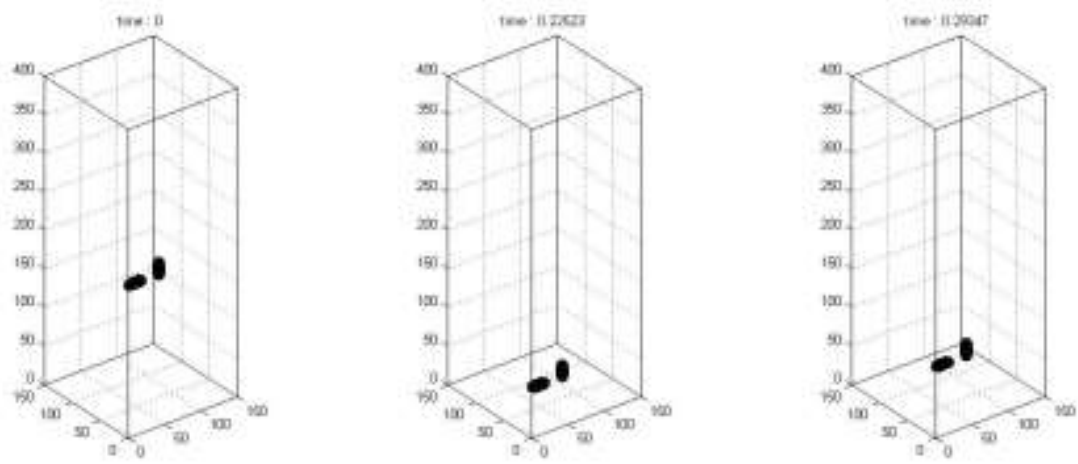

Figure 5: Test of particles-wall contact by falling particles to the bottom of the bed

Every particle hits the bottom floor at the same time and the same velocity. The velocity that the particles hit the bottom floor is about $1.77 \mathrm{~m} / \mathrm{s}$ which is the same value from the calculation. The forces acting on the particle are only gravitation force and force from the bottom wall contact. There is no movement in $\mathrm{X}-\mathrm{Z}$ directions and also for the rotation. This results from the forces acting on this two particle pass through their center of mass.
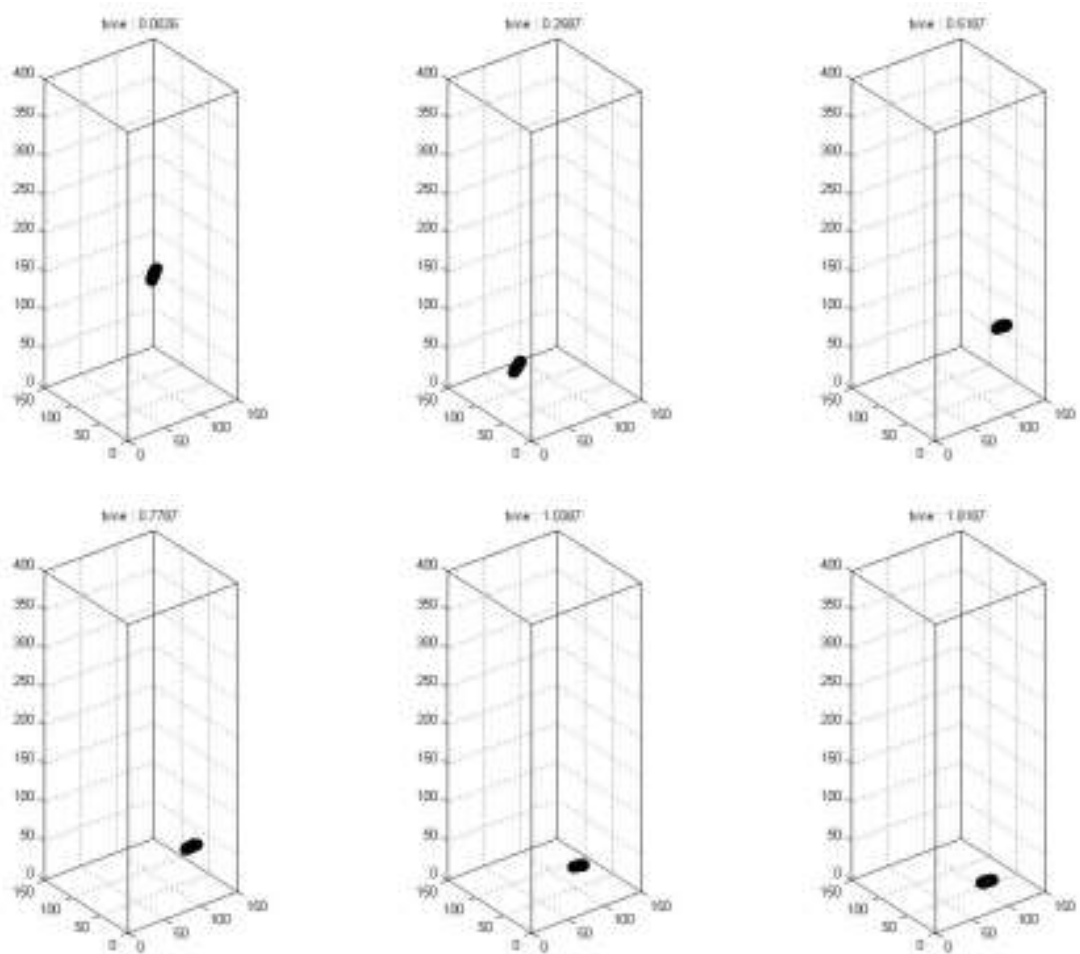

Figure 6: Test of particles-wall contact by falling particles to the bottom of the bed with 60 degrees to the X-Y plane

Fig 6.shows the particle laid in 60 degrees with $\mathrm{X}-\mathrm{Z}$ plane to study the effect of force and rotation. The velocity before the collision is the same as the previous study. The forces acting on the particle are gravitation force and force from the wall contact. After collision, the particle bounces and moves in X-Y-Z directions, then it hits the left wall, the height after collision is about $140 \mathrm{~mm}$. This phenomenon occurs from the forces acting on the particle do not pass the center of mass causing the velocity in both translation and rotation. Moreover, the simulation result is compared with the calculation of direct motion with constant accelerate. The accuracy of between the simulation and the physical calculation is acceptable.

\subsubsection{Particle-Particle Contact}

Particle-particle contact can be divided into 2 cases; the particles contact in the direction along the center line between the particles and the general directions. The viscous damping force is included, but the gravitation force is not 
accounted for. Therefore, the forces in this study are the particle-particle contact force and particle-wall contact force. These forces can be divided into contact force and viscous damping in the normal and tangential direction. Fig.7 shows two spherocylinder particles with different orientation collide through the center line in X-direction. The first particle moves in X-direction with velocity $0.8 \mathrm{~m} / \mathrm{s}$ while the second particle is at rest at time 0 second. The angular velocities in $\mathrm{X}, \mathrm{Y}$ and Z-direction are $0.0 \mathrm{rad} / \mathrm{s}$ for both particles.
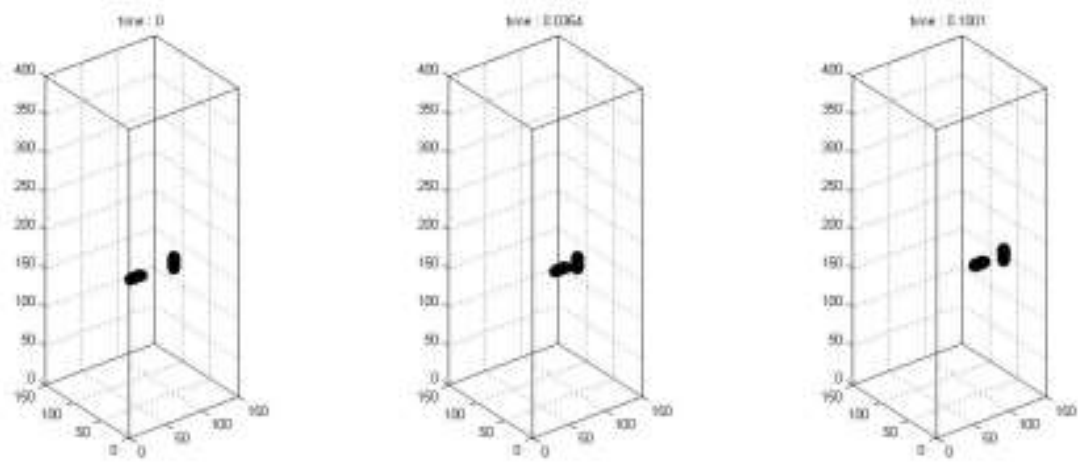

Figure 7: Test of particle-particle collision along the center line

After the particles hit each other, the velocities in X-direction are $0.14 \mathrm{~m} / \mathrm{s}$ for first particle and $0.66 \mathrm{~m} / \mathrm{s}$ for the other. There is only translation velocity but no angular velocity for both particles since the collision takes place along the center line.
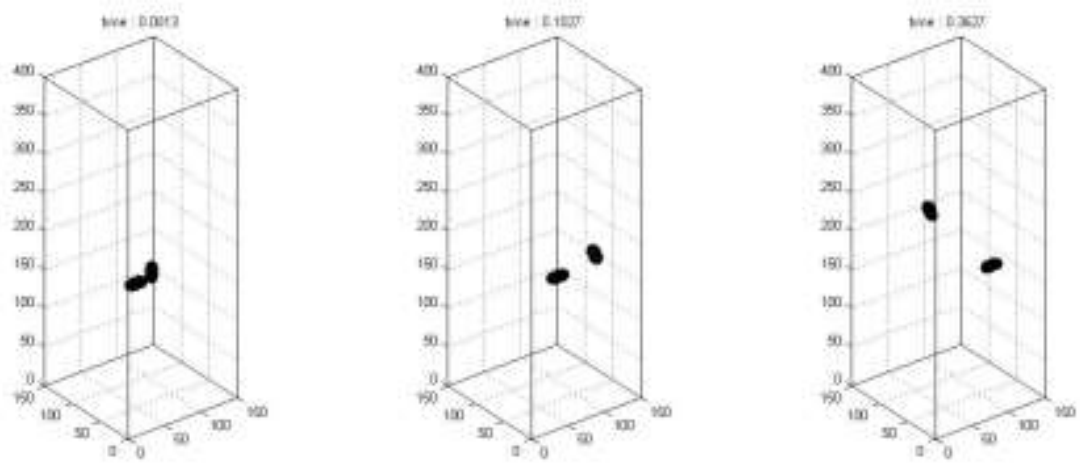

Figure 8: Test of particle-particle collision in general direction

Fig. 8 shows two spherocylinder particles with different orientation collide through the endpoint of another particle. The first particle laid horizontally moves in X-direction with velocity $0.8 \mathrm{~m} / \mathrm{s}$ while the second particle is at rest at time 0 second. The angular velocities in X, Y and Z-direction are $0.0 \mathrm{rad} / \mathrm{s}$ for both particles. After the collision occurred, the velocities in X-direction are $0.15 \mathrm{~m} / \mathrm{s}$ for the first particle and $0.62 \mathrm{~m} / \mathrm{s}$ for the other. Also, the second particle has the velocity in Y-direction equal to $0.043 \mathrm{~m} / \mathrm{s}$. There is no angular velocity for the first particle since the collision takes place along the center line. For the second particle, the angular velocity after the collision is about $4.19 \mathrm{rad} / \mathrm{s}$ in Z-direction. The difference between these two cases observed forms the visualization is that the second particle would change its direction after the collision. It will move in $\mathrm{X}, \mathrm{Y}$ and Z-direction instead of X-direction only.

\subsubsection{Particle-Fluid Contact}

This section shows the particle motion under the presence of the fluid motion. Two particles are set at $100 \mathrm{~mm}$ above the bottom wall and the air inlet orifices. The air from orifices is fed in sequence every 0.5 seconds. The orifices are fed with the air at the dry bulk temperature and room temperature to study the effect of momentum and heat transfer. As shown in the Fig.9; before 0.5 seconds, both gravitation force and fluid drag forces act on the first particle but only gravitation force effects in the different particle motion. Also, the first particle will be heated up by hot air. 

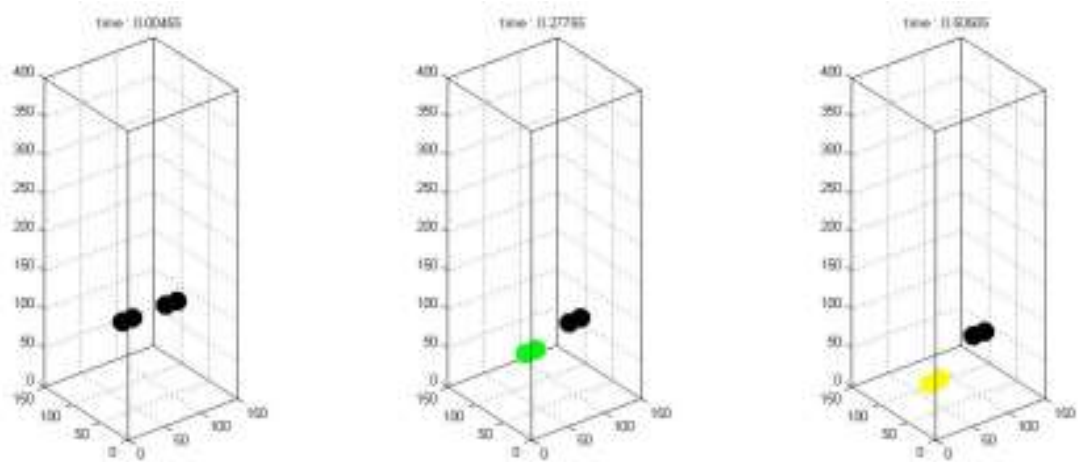

Figure 9: Test of particle-fluid contact for momentum and heat transfer

- $<299.15 \mathrm{~K}$

$307.15-310.15 \mathrm{~K}$
- $299.15-302.15 \mathrm{~K}$

- $310.15-313.15 \mathrm{~K}$
- $302.15-304.15$ • 304.15-307.15

- > 313.15

\subsection{Random Packing of Particles}

In this step, the particles would be randomly placed inside the bed at the beginning of the simulation. Then, the particles fall by gravitation force, and particles would pack at the bottom of the bed. Each particle is shown with different colors to present the random motion of particles. This initial position of each particle is required to study the momentum, heat and mass transfer of spherocylinder particle.
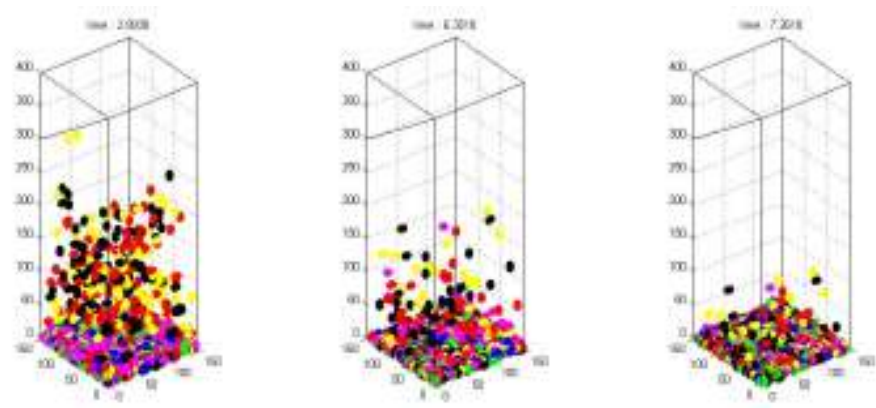

Figure 10: an Initialization step

\subsection{The Study of Momentum, Heat and Mass Transfer}

This part can be divided into two sections; the First section illustrates the effect of pulsation of inlet gas velocities on the drying rate and the second section is to study the impact of bed configuration. In the first section, the superficial gas velocities are 3.0, 6.0, 10.0 and $13.0 \mathrm{~m} / \mathrm{s}$ and the results are shown in Fig.11 - 12 for the superficial gas velocities 10.0 and $13.0 \mathrm{~m} / \mathrm{s}$, respectively. The air is fed in sequence every 0.5 seconds to each orifice. Each particle in the bed is represented as a pair of the point with a different color to indicate different particle temperature.

At superficial gas velocity 3.0 and $6.0 \mathrm{~m} / \mathrm{s}$, the temperature of a particle slightly changes except the area near the orifices, this comes from gas could not flow through the particles well so gas-solid contact was low as well as the heat transfer coefficient. Most of the particles move slightly in X-Z direction. At the dead zone, gas does not flow through the bed so the particle is not moved and less heat transfer considered in this zone. Increasing superficial velocity to 10.0 and $13.0 \mathrm{~m} / \mathrm{s}$ significantly improves the heat transfer, and the particle motion can be observed clearly. It can be noticed from the simulation results that when the particle is heated to the wet-bulb temperature of the gas, the drying in constant rate period is started. At this period, the temperature of the particle is constant. The particle reaches the wet-bulb temperature faster after increasing the superficial velocity. When the fluid drag force of gas had more than the total force acting on the particles, then the particles are bounced. This can be observed at a superficial velocity more than $10.0 \mathrm{~m} / \mathrm{s}$, and spouted behavior of the bed can also be seen obviously near the orifices. At high superficial velocity, the particle temperature distribution and heat transfer between particles increases resulting from the improvement particle motion. 

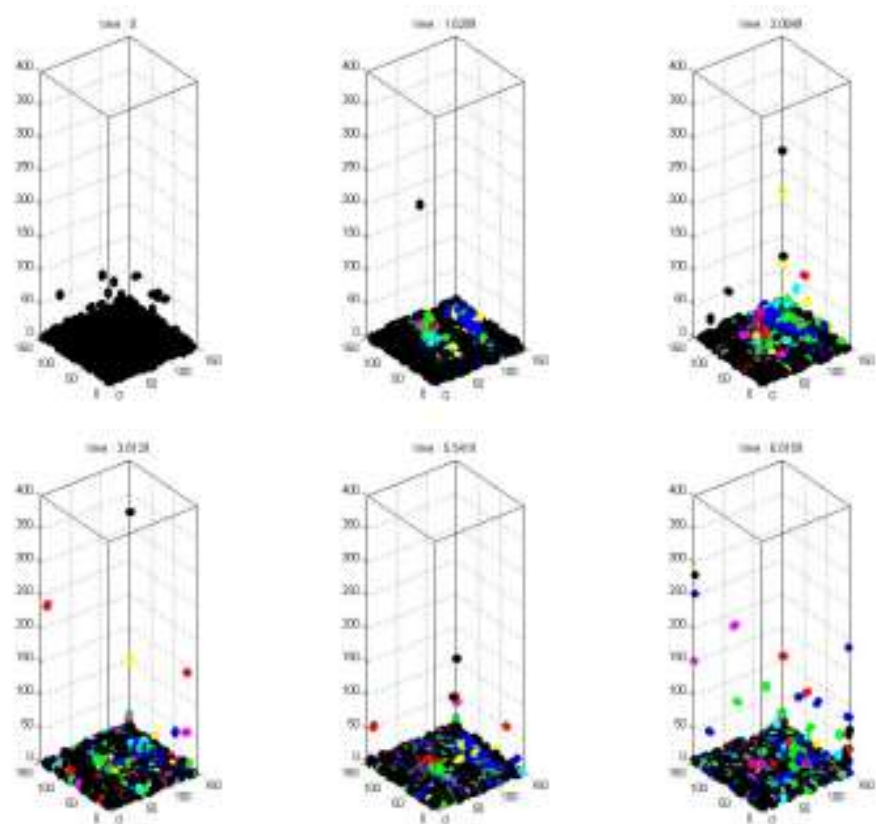

Figure 11: Particle motion at $\mathrm{u}_{0}=10.0 \mathrm{~m} / \mathrm{s}$
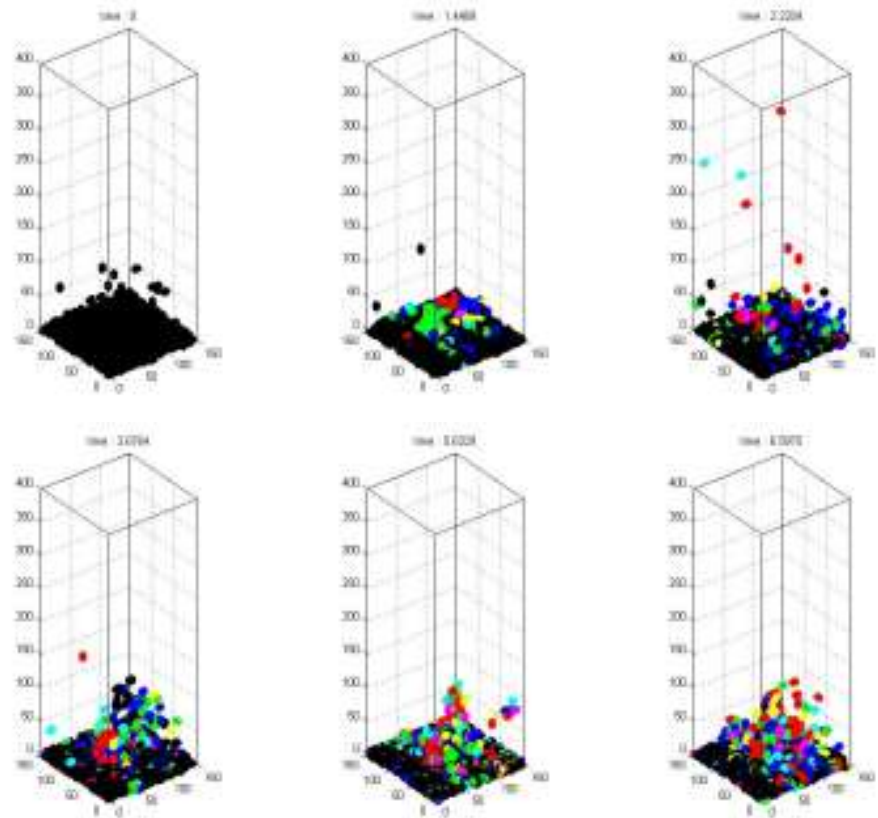

Figure 12: Particle motion at $\mathrm{u}_{0}=13.0 \mathrm{~m} / \mathrm{s}$

- $<299.15 \mathrm{~K}$

$307.15-310.15 \mathrm{~K}$
- 299.15-302.15 K

- $310.15-313.15 \mathrm{~K}$
- 302.15-304.15• 304.15-307.15

- > 313.15

After the effect of superficial gas velocity is studied, the effect of bed configuration on the particle motion and heat and mass transfer will be performed. The feed inlet form previous section will be modified from 2 feed inlets to 1 feed inlet and 4 feed inlets, respectively. The total area of feed inlet remains constant. This part focuses only on the particle motion, heat and mass transfer of spherocylinder particles at superficial gas velocity $13.0 \mathrm{~m} / \mathrm{s}$ since the spouted bed behavior is significantly observed at the superficial gas velocity above $13.0 \mathrm{~m} / \mathrm{s}$. 

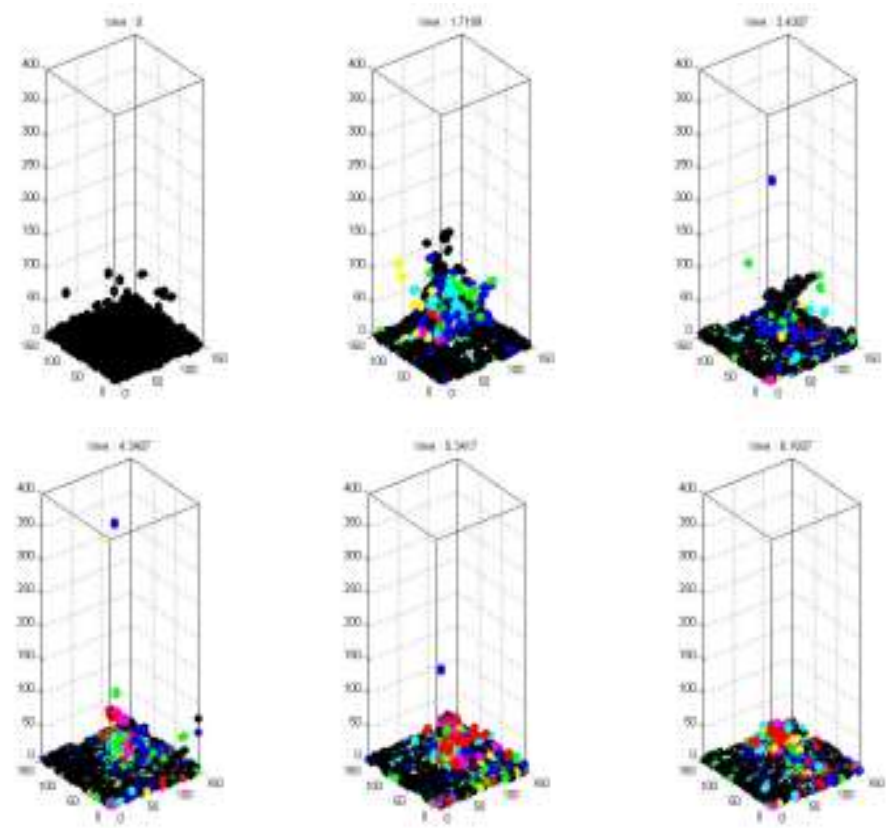

Figure 13: Particle motion at $\mathrm{u}_{0}=13.0 \mathrm{~m} / \mathrm{s}$ ( 1 Feed inlet)
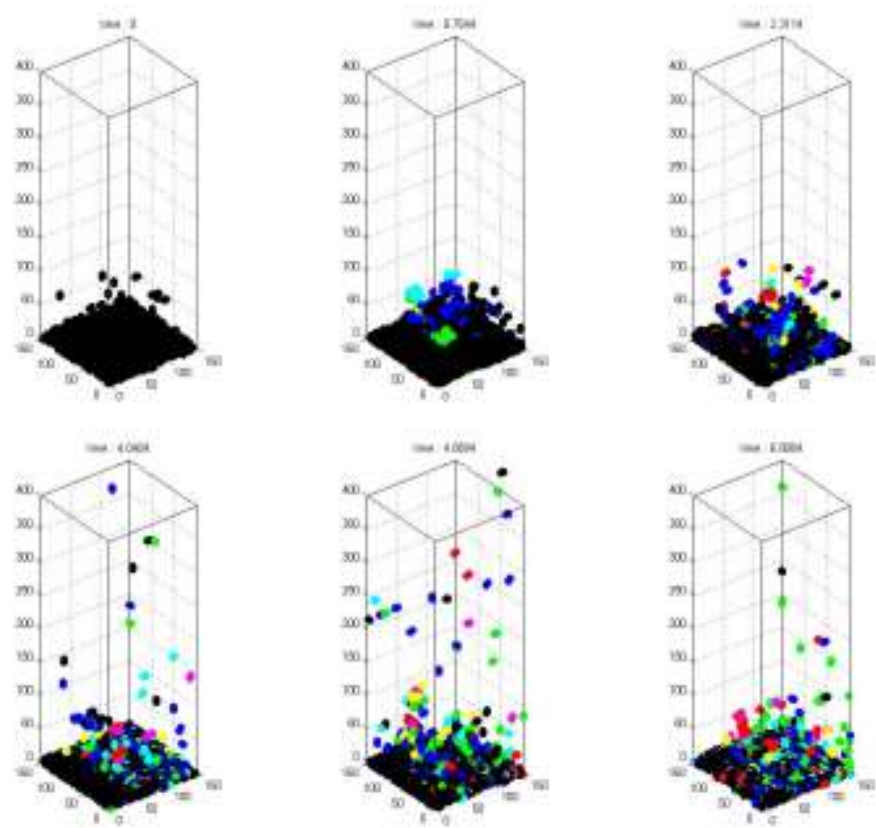

Figure 14: Particle motion at $\mathrm{u}_{0}=13.0 \mathrm{~m} / \mathrm{s}$ (4 Feed inlets)

- $<299.15 \mathrm{~K}$

307.15-310.15 K
- $299.15-302.15 \mathrm{~K}$

- $310.15-313.15 \mathrm{~K}$
- 302.15-304.15 304.15-307.15

- > 313.15

At the time when the orifices have released the gas flow, the particles above the feed inlet are driven by the fluid drag force and interparticle force to behave as the spouted bed. After the gas flow disappeared, all particles fall by the gravitation force. The difference between these bed configurations causes a significantly different movement of particles. More feed inlets improve the particle motion increasing heat and mass transfer. As shown in Fig.12 and 14, more feed inlets improve the particle temperature distributions at the same superficial gas velocity.

\subsection{Drying of Solids}




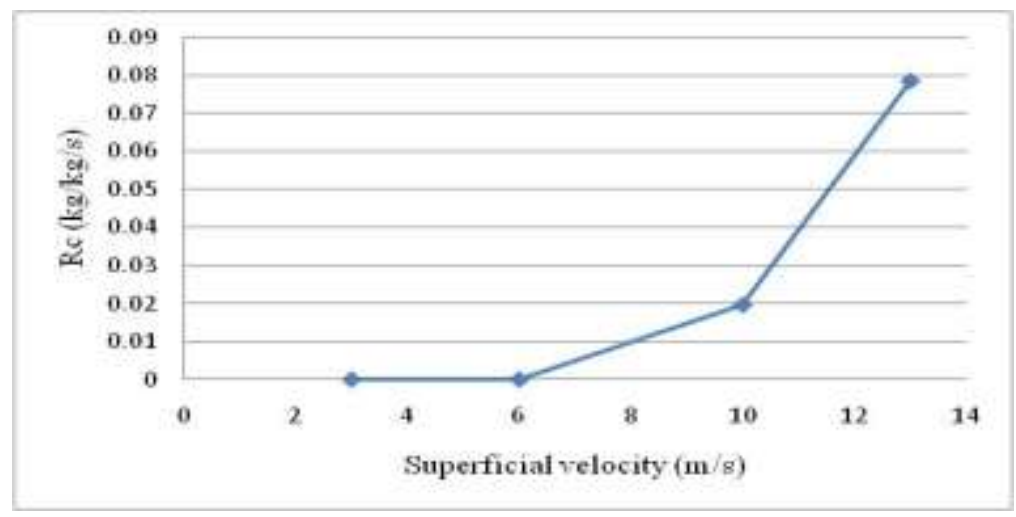

Figure 15: Effect of superficial velocity on the drying rate of particles ( 2 feed inlets)

From Fig.15, there is no drying rate occurred at superficial velocity 3.0 and $6.0 \mathrm{~m} / \mathrm{s}$ at the time which is interested in. There is only momentum and heat transfer at these superficial gas velocities. For superficial gas velocity $10.0 \mathrm{~m} / \mathrm{s}$, the particles reach to the wet-bulb temperature of the inlet air $(313.15 \mathrm{~K})$ at the time about $5.0 \mathrm{sec}$ while they reach the wetbulb temperature at the time about $4.5 \mathrm{sec}$ in the case of superficial $13.0 \mathrm{~m} / \mathrm{s}$. The particles inside the bed reach the wetbulb temperature faster after increasing the superficial velocity. Also, the drying rate of particles at a constant rate period also increases after increasing the superficial gas velocity. Therefore, it can be summarized that the heat transfer inside of spherocylinder particles increases with the increasing of superficial velocity due to the improvement of spouting behavior.

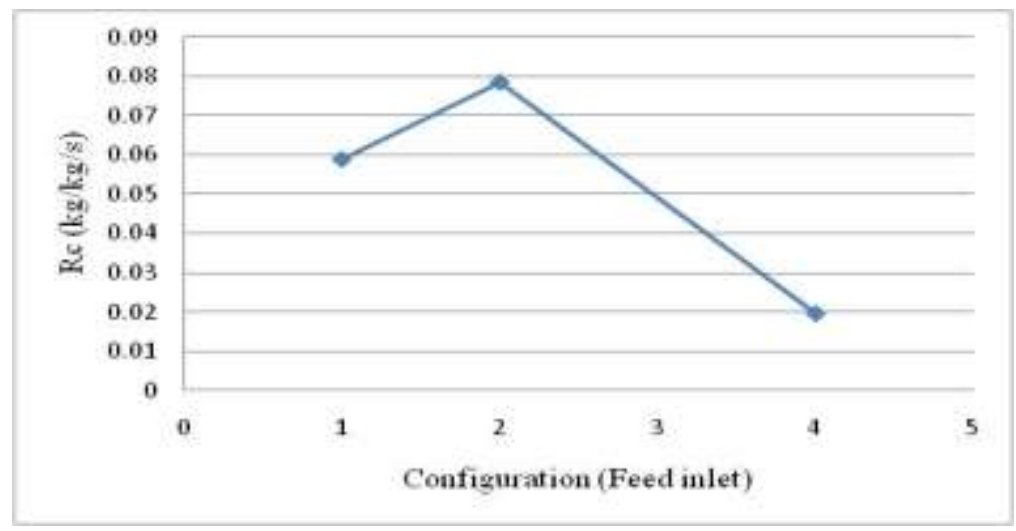

Figure 16: Drying curve of particles at $\mathrm{u}_{0}=13.0 \mathrm{~m} / \mathrm{s}$

From Fig.16, the best drying rate occurs at number feed inlet equal to 2 . At number feed inlet equal to 2, the particles in the bed reach to the wet-bulb temperature of the inlet air $(313.15 \mathrm{~K})$ at the time about $4.5 \mathrm{sec}$ while they reach the wetbulb temperature at the time about $6.0 \mathrm{sec}$ and $6.3 \mathrm{sec}$ in the cases of number of feed inlet 1 and 4 , respectively. This can be summarized that even though the increase of feed inlet will provide more particle motion and particle temperature distribution; it decreases the area per feed inlet and time for air to be fed through each nozzle before being fed to the next nozzle resulting in slower drying rate.

\section{CONCLUSIONS AND RECOMMENDATIONS}

\subsection{Conclusions}

In this work, the discrete element simulation scheme is performed to handle the spherocylinder particle motion in three dimensions. This work uses a combined approach of discrete element method and computational fluid dynamics (DEM-CFD) to calculate the momentum, heat and mass transfer. The program is developed using standard C-language, and the results are visualized in an animation style using MATLAB ${ }^{\mathrm{TM}}$. The calculation of the neighborhood particles around a particle interested in within boundaries and contact list is obtained to reduce the computational time. Two thousands of particles are studied in the particle motion, the effect of superficial gas velocity and the bed configuration on the particle temperature distribution and drying rate. In the first step, the program is tested to determine the realistic of particle motion. Then two thousands of spherocylinder particles are left to free fall by the gravity to make the initialization step. In the later stage, the hot air is fed into the bed with different superficial gas velocity (3.0, 6.0. 10.0 and $13.0 \mathrm{~m} / \mathrm{s}$ ) and the configuration of the bed is also modified. The results are reasonably realistic in particle motion, particle temperature distribution and the drying rate. The particles near the orifices are heated up rapidly while other particles around and in the edges of the chamber heat up slower. At low superficial gas velocity, the particles inside the 
bed could not be spouted but smoothly circulated resulting in low heat transfer. Increasing of superficial gas velocity to 10.0 and $13.0 \mathrm{~m} / \mathrm{s}$ significantly improves the heat transfer, particle motion, and drying rate. For the effect of bed configuration, the number of feed inlet is varied to 1 and 4 feed inlets by maintaining the total area of feed inlet and the superficial gas velocity. The results demonstrate that the increasing number of feed inlet improves particle movement and particle temperature distribution. However, the drying rate of 4 gas inlets decreases from the drying rate of 2 feed inlets. This phenomenon comes from the air fed through each nozzle has a short period before the air is fed through the next nozzle.

\subsection{Recommendations}

This program is not completed correctly since there are time limitation and difficult for programming the code. This programming can be simulated only at a time below 8 seconds. This may affect the accuracy of drying rate determination from the unsteady state condition. Therefore, the validity of this programming and drying rate should certainly be further performed. Moreover, the collision between particle-particle in parallel direction should be further developed.

\section{ACKNOWLEDGMENT}

We would like to thank Mr. Pakin Chuenyuenyong whose original 3D programs of sphere particles were used as an education model of DEM and CFD. We want to pay my gratitude to ChEPS, King Mongkut's University of Technology Thonburi for the great opportunity.

\section{REFERENCES}

[1] Mathur, K.B. and Epstein, N., Spouted Beds, Academic Press (1974), New York.

[2] Cundall, P.A. and Strack, O.D.L., A Discrete Numerical Model for Grannular Assemblies, Geotechnique, Vol. 29 (1979), No. 1, 47-65.

[3] Abreu, C.R.A., Tavares, F.W., Cartier, M., Influence of particle shape on the packing and the segregation of spherocylinders via Monte Carlo simulations, Powder Technology, Vol. 134 (2003), 167-180.

[4] K.B. Mathur and P.E. Gishler, A technique for contacting gases with coarse solid particles, AIChE. Journal 1 (1955), 157-164.

[5] Pournin, L., Webber, M., Tsukahara, M. and Ferrez, J.A., Three-dimensional distinct element simulation of spherocylinder crystallization, Granular Matter, Vol 7 (2005), 119-126.

[6] Ergun, S., Chemical Engineering Progress, Vol. 48 (1952), No. 2, 123-232.

[7] Wen, C.Y. and Yu, Y.H., Chemical Engineering Progress, Series 62, Vol. 62 (1996), 143-156.

[8] Fan. L., Mao, Z. and Yang, C., Experiment on Settling of Slender Particles with Large Aspect Ratio and Correlation of the Drag Coefficient, Industrial \& Engineering Chemistry Research, Vol. 43 (2004), 7664-7679.

[9] Li J. and Mason D.J., A Computational Investigation of Transient Heat Transfer in Pneumatic Transport of Granular Particles, Powder Technology, Vol. 112 (2000), 273-282. 